\title{
Engineering quantum magnetism in one-dimensional trapped Fermi gases with $p$-wave interactions
}

\author{
Lijun Yang, ${ }^{1}$ Xiwen Guan, ${ }^{2,3}$ and Xiaoling Cui ${ }^{1, *}$ \\ ${ }^{1}$ Beijing National Laboratory for Condensed Matter Physics, Institute of Physics, Chinese Academy of Sciences, Beijing 100190, China \\ ${ }^{2}$ State Key Laboratory of Magnetic Resonance and Atomic and Molecular Physics, Wuhan Institute of Physics and Mathematics, \\ Chinese Academy of Sciences, Wuhan 430071, China \\ ${ }^{3}$ Department of Theoretical Physics, Research School of Physics and Engineering, Australian National University, \\ Canberra ACT 0200, Australia \\ (Received 2 December 2015; published 31 May 2016)
}

\begin{abstract}
The highly controllable ultracold atoms in a one-dimensional (1D) trap provide a new platform for the ultimate simulation of quantum magnetism. In this regard, the Néel antiferromagnetism and the itinerant ferromagnetism are of central importance and great interest. Here we show that these magnetic orders can be achieved in the strongly interacting spin-1/2 trapped Fermi gases with additional $p$-wave interactions. In this strong-coupling limit, the 1D trapped Fermi gas exhibits an effective Heisenberg spin $X X Z$ chain in the anisotropic $p$-wave scattering channels. For a particular $p$-wave attraction or repulsion within the same species of fermionic atoms, the system displays ferromagnetic domains with full spin segregation or the antiferromagnetic spin configuration in the ground state. Such engineered magnetisms are likely to be probed in a quasi-1D trapped Fermi gas of ${ }^{40} \mathrm{~K}$ atoms with very close $s$-wave and $p$-wave Feshbach resonances.
\end{abstract}

DOI: 10.1103/PhysRevA.93.051605

Introduction. For several decades quantum magnetism is one of the central research fields in condensed matter physics [1]. In recent years, the ultracold atomic gases provide an ideal platform for the exploration of quantum magnetism owing to the high controllability of the interaction and the geometry [2,3]. In particular, the Néel antiferromagnetism (AFM) in lattices and the itinerant ferromagnetism (FM) in continuum have been attracting great attention in both theory and experiment with ultracold atoms. However, the long-range Néel AFM requires sufficiently low temperature associated with the superexchange coupling in lattices, which is beyond what the current cooling technique can achieve so far. In fact, only the short-range AFM correlation was successfully probed recently [2,3]. The exploration of the itinerant FM in the repulsive branch of three-dimensional (3D) trapped Fermi gases was unsuccessful, where the possible ferromagnetic state was impeded by severe atom losses when the system approaches the resonance regime [4,5]. There have been various proposals for realizing itinerant FM in ultracold atoms, for instance, by using the mass-imbalance [6-10], external potentials [11-14], SU(N) Fermi gases [15], spinor bosons [16], or multiorbital [17] systems. Yet to observe the itinerant FM is still quite challenging in realistic experiments.

In this context, the one-dimensional (1D) strongly interacting atomic gases offer an alternative while promising opportunity for the simulation of quantum magnetism in cold atoms; see a recent review [18]. The hidden magnetic spin-chain structures for the strongly interacting bosons and fermions provide insight into the simulation and understanding of FM and AFM orderings [12,13,16,19-25]. Given the impenetrable feature of particles in a strong-coupling limit [26-29], an underlying "lattice" spin chain can be supported with the site index given by the order of particles distributed

\footnotetext{
*xlcui@iphy.ac.cn
}

in the spatial space (see Fig. 1). For a large but finite $s$-wave coupling, the 1D trapped Fermi gas is well decoupled into the charge and spin degrees of freedom with an effective Heisenberg spin chain in the spin sector [12,13,16,22-25]. In this effective spin chain, the nearest-neighbor superexchange interaction is fully determined by the particle collision in the overlap region of their density distributions.

Such a system allows one to study quantum magnetism in the absence of lattice potentials [30]. In particular, the atom loss therein can be greatly suppressed by the hard-core interaction [31,32], contrary to the large atom loss in a 3D repulsive gas approaching the resonance [5].

The effective spin chain can well explain the magnetic correlations in the strongly interacting spin-1/2 trapped fermions [11,33-36]. In these works, the AFM correlation for repulsive fermions [33] and the FM transition at the point of full fermionalization [11] were discussed. Very recently, such an AFM correlation has been experimentally investigated in a small cluster system of 1D trapped fermions [37].

In this work, we propose to engineer both the Néel AFM and the itinerant FM in a strongly $s$-wave interacting spin-1/2 Fermi gas with additional $p$-wave interactions in a 1D harmonic trap. We find a general effective Heisenberg $X X Z$ model induced by the anisotropic $p$-wave interactions. This model is expected to result in a rich quantum magnetism by adjusting $p$-wave interaction strengths in different (spin-triplet) channels. In particular, we consider the $p$-wave interaction within one fermion species, as is realizable in the spin-1/2 Fermi gas of ${ }^{40} \mathrm{~K}$ atoms with close $s$ - and $p$-wave Feshbach resonances [38]. We show that by switching on a weak $p$-wave attraction, the ground state of strongly ( $s$-wave) interacting fermions in the 1D trap gradually form the FM domains with full spin segregation, while a weak $p$-wave repulsion will induce the Néel long-range AFM order.

The model. We start from the Hamiltonian for 1D trapped spin-1/2( $\uparrow, \downarrow)$ fermions with both $s$ - and $p$-wave interactions, 


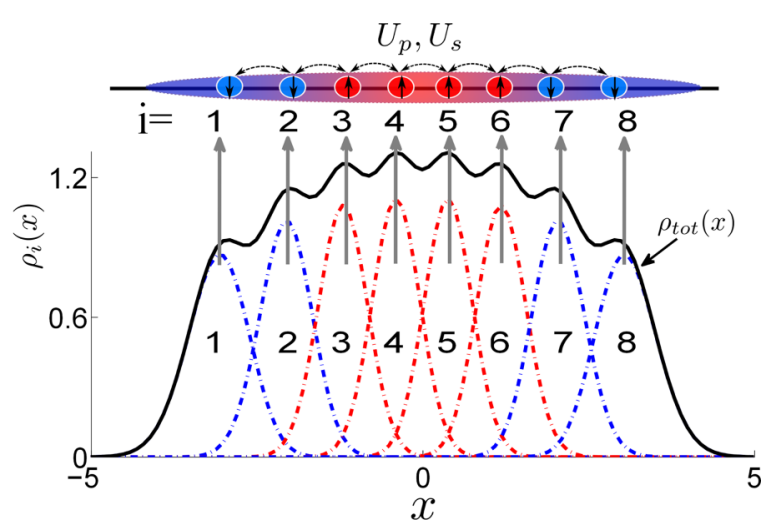

FIG. 1. Density distribution of fermionalized atoms in a harmonic trap. Black solid line is the total density; blue dashed line is for particle at order number $i=1,2, \ldots N(N=8)$ from left to right. $\rho_{i}$ and $x$ are, respectively, in units of $1 / a_{T}$ and $a_{T}\left(a_{T}=1 / \sqrt{m \omega_{T}}\right.$ is the confinement length). Each order number can be effectively mapped to the site index in a spin chain, with nearest-neighbor coupling determined by the finite $s$ - or $p$-wave interactions. A weak $p$-wave attraction between spin- $\uparrow$ atoms will drive the system to itinerant ferromagnetism with full spin segregation (see the spin distribution along the tube).

$H=H_{0}+U_{s}+U_{p}($ we set $\hbar=1)$ :

$$
\begin{gathered}
H_{0}=\sum_{i}\left(-\frac{1}{2 m} \frac{\partial^{2}}{\partial x_{i}^{2}}+\frac{1}{2} m \omega_{T}^{2} x_{i}^{2}\right), \\
U_{s}=-\frac{2}{m a_{1 D}} \sum_{i, j} \delta\left(x_{i \uparrow}-x_{j \downarrow}\right), \\
U_{p}=\sum_{i<j} \sum_{M=0, \pm 1}-\frac{2 l_{p}^{(M)}}{m} \partial_{x_{i j}} \delta\left(x_{i j}\right) \partial_{x_{i j}} P_{M}(i, j),
\end{gathered}
$$

with $x_{i j}=x_{i}-x_{j}$. Here $U_{s}$ and $U_{p}$ denote the $s$ - and $p$-wave interactions with scattering lengths $a_{1 D}$ and $l_{p}^{(M)}$, respectively, in the spin-singlet and spin-triplet (with total magnetization $M=0, \pm 1$ ) channels, and $P_{M}$ is the according spin-projection operator in the triplet channel. Note that for obtaining Eq. (3) we have limited ourselves to the weak $p$-wave interactions [39].

For the homogenous gas $\left(\omega_{T}=0\right)$, the two-body scattering matrix does not comprise the Yang-Baxter equation [40]. Thus this model is not integrable in the presence of both s- and p-wave interactions except for a few particular cases, i.e., for $l_{p}^{(M)}=0$ the model reduces to the Yang-Gaudin model, whereas for $a_{1 D}=0$ and $l_{p}^{(0)}=l_{p}^{( \pm 1)}$ it reduces to the $\mathrm{SU}(2)$ spinor Bose gas with a ferromagnetic ground state [19]; also see a recent discussion in [41]. In the following discussion we will consider the quasi-1D trapped Fermi gas with a strong $s$-wave interaction and a weak $p$-wave interaction, i.e., $\left|a_{1 D}\right|,\left|l_{p}^{M}\right|$ are much smaller than the mean particle distance. The physics in this regime can be well deduced from the fermionalized limit in the framework of perturbation theory, a basic idea for the construction of an effective model as follows.

The effective spin chain. At the point of full fermionization $\left(a_{1 D}=l_{p}^{(M)}=0\right)$, the ground state of the system is highly degenerate and follows the form of

$$
\Psi_{\xi}=\phi_{F}\left(x_{1}, x_{2}, \ldots, x_{N}\right)\left\langle\left\{x_{i}\right\} ;\left\{\mu_{i}\right\} \mid \vec{\xi}\right\rangle
$$

where $\phi_{F}=\frac{1}{\sqrt{N !}} D\left(x_{1}, x_{2}, \ldots, x_{N}\right)$ is the Slater determinant composed by the lowest $N$ level of eigenstates of $H_{0}$ and $\left\{\mu_{i}\right\}$ denote the spins of the atoms. In the above equations $|\vec{\xi}\rangle$ is the spin-ordered state,

$$
\left\langle\left\{x_{i}\right\} ;\left\{\mu_{i}\right\} \mid \vec{\xi}\right\rangle=\sum_{P} \theta\left(x_{P_{1}}<x_{P 2}<\cdots<x_{P_{N}}\right) \prod_{i} \delta_{\xi_{i}, \mu_{P_{i}}}
$$

describing a sequence of spins $\xi_{1}, \xi_{2}, \ldots, \xi_{N}$ placed in order from left to right in the coordinate space. By considering different spin orders $\vec{\xi}$, one can cover all the degenerate ground states.

In the vicinity of full fermionization ( $\operatorname{small} a_{1 D}, l_{p}^{(M)}$ ), the particles sitting at the neighbors can collide and exchange their spins. This process can be well described by an effective spin-chain Hamiltonian by mapping the orders of particles into site indexes on a spin chain. In the absence of $p$ wave $\left(l_{p}^{(M)}=0\right)$, it is the Heisenberg model associated with $s$-wave interaction $[12,13,22,23,25]$, namely,

$$
H_{\mathrm{eff}}^{s}=-\frac{m a_{1 D}}{2} \sum_{l} J_{l}\left(\mathbf{s}_{l} \cdot \mathbf{s}_{l+1}-\frac{1}{4}\right)
$$

where $s_{l}^{\alpha}(\alpha=x, y, z)$ is the spin operator for an atom at site $l$, and the site-dependent nearest-neighbor coupling is given by

$$
J_{l}=\left.\frac{N !}{2}\left(\frac{2}{m}\right)^{2} \int d \mathbf{x}\left|\frac{\partial \phi_{F}}{\partial x_{i j}}\right|_{x_{i j}=0}\right|^{2} \theta\left(\cdots<x_{i}=x_{j}<\cdots\right),
$$

where $d \mathbf{x}=\prod_{i} d x_{i}$, and $x_{i}\left(=x_{j}\right)$ is placed in the $l$ th order in the $\theta$ function.

In the presence of a weak $p$-wave interaction, we can derive an effective spin-chain Hamiltonian based on the first-order perturbation theory. Given a general ansatz for the many-body wave function

$$
\Psi\left(\left\{x_{i}\right\} ;\left\{\mu_{i}\right\}\right)=\phi_{F}\left(\left\{x_{i}\right\}\right) \sum_{\xi} a_{\xi}\left\langle\left\{x_{i}\right\} ;\left\{\mu_{i}\right\} \mid \vec{\xi}\right\rangle,
$$

we can express the energy function produced by $p$-wave interaction as $E\left(\left\{a_{\xi}\right\}\right)$. By mapping the spin order number to the site index on a chain, we arrive at the following effective Hamiltonian which will reproduce the same energy functional $E\left(\left\{a_{\xi}\right\}\right)$ :

$$
H_{\mathrm{eff}}^{p}=\sum_{M=0, \pm 1} \frac{m l_{p}^{(M)}}{2} \sum_{l} J_{l} P_{M}(l, l+1),
$$

where $P_{M}(l, l+1)$ is the projection operator of the neighboring-site atoms into a spin triplet with the magnetization $M$ along $z$ direction. Remarkably, here the coupling $J_{l}$ follows the same expression as in the $s$-wave case [Eq. (7)], despite the completely different forms between the $s$ - and $p$-wave contact potentials [Eqs. (2) and (3)]. The underlying physics for the similar structure of $H_{\mathrm{eff}}^{s}$ and $H_{\mathrm{eff}}^{p}$ share the same spirit as the boson-fermion duality [42], i.e., the bosons with a strong $s$-wave interaction $g$ are equivalent to the fermions 


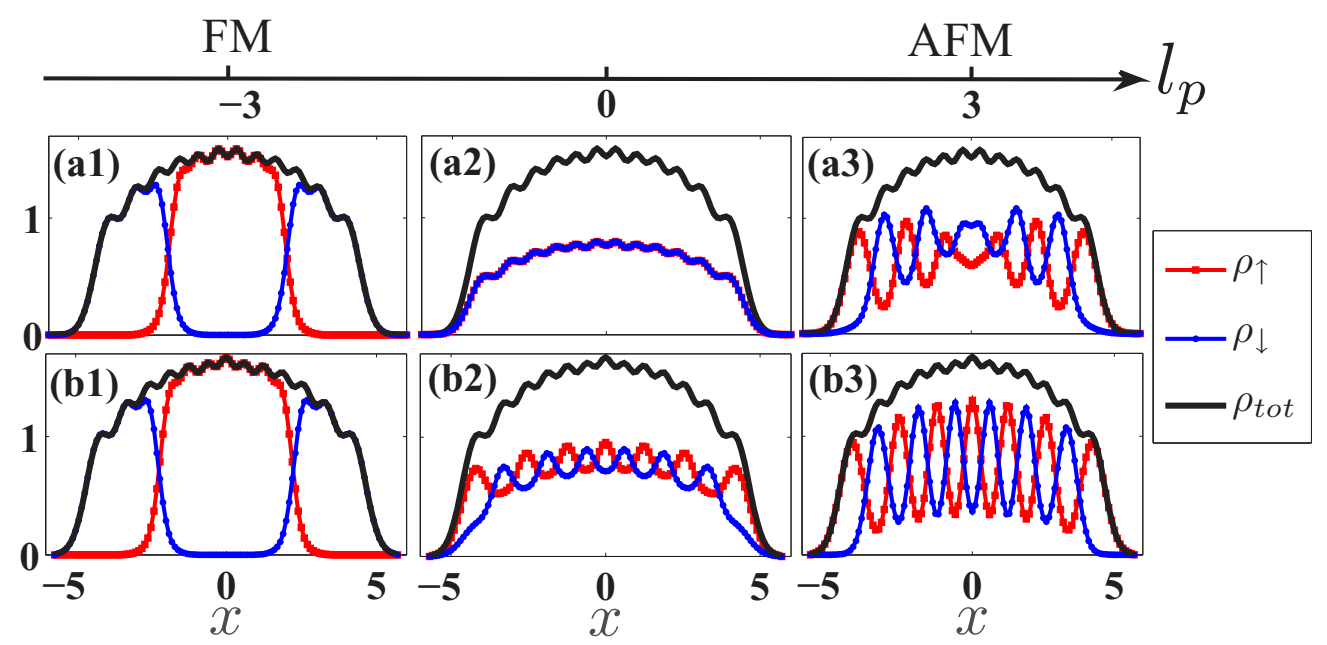

FIG. 2. Magnetic orders induced by $p$-wave interaction between spin- $\uparrow$ atoms. Upper panel (a1-a3) and lower panel (b1-b3) are, respectively, the spin density distributions of $6 \uparrow+6 \downarrow$ and $7 \uparrow+6 \downarrow$ systems. Three values of $l_{p}$ (in unit of $-a_{1 D}$ ) are considered: -3 (a1,b1), 0 (a2,b2), and $3(\mathrm{a} 3, \mathrm{~b} 3) . \rho_{\sigma}(x)$ and $x$ are respectively in units of $1 / a_{T}$ and $a_{T} .\left(a_{T}=1 / \sqrt{m \omega_{T}}\right.$ is the confinement length.)

with a weak $p$-wave interaction $1 / g$. This mechanism can be generalized to the " $s$ " - $p$ " duality in the spin- $1 / 2$ fermionic system in this work. Here $s$ and $p$ refer to partial-wave scattering channels.

In fact, $H_{\mathrm{eff}}^{p}$ [Eq. (9)] represents the Heisenberg spin $X X Z$ model with an effective magnetic field (up to a constant shift):

$$
H_{\mathrm{eff}}^{p}=\sum_{l} j_{l}^{\perp}\left(s_{l}^{x} s_{l+1}^{x}+s_{l}^{y} s_{l+1}^{y}\right)+j_{l}^{z} s_{l}^{z} s_{l+1}^{z}+h_{l}\left(s_{l}^{z}+s_{l+1}^{z}\right),
$$

with $j_{l}^{\perp}=m J_{l} l_{p}^{(0)} / 2, \quad j_{l}^{z}=m J_{l}\left(l_{p}^{(1)}+l_{p}^{(-1)}-l_{p}^{(0)}\right) / 2, \quad h_{l}=$ $m J_{l}\left(l_{p}^{(1)}-l_{p}^{(-1)}\right) / 4$. The fundamental physics of the Heisenberg spin $X X Z$ model can be found in [43]. For the isotropic $p$-wave case with all equal $l_{p}^{(M)}$, Eq. (10) reproduces the AFM or FM Heisenberg model for positive or negative $l_{p}^{(M)}$. For the anisotropic case of $l_{p}^{(M)}$, which may break both the spin-rotation and the time-reversal symmetry, the system exhibits a versatile type of magnetic orders depending on the sign and relative strength of $l_{p}^{(M)}$ in different $p$-wave scattering channels.

In the rest of the paper, we will consider a realistic case of $p$-wave interaction in ${ }^{40} \mathrm{~K}$ Fermi gases, which only exists between one species of fermions (suppose spin $\uparrow$ ) [38]. We will show that despite this simple $p$-wave interaction, the system can display rich and nontrivial magnetic ordering. Our analysis is based on the full effective Hamiltonian $H_{\mathrm{eff}}=H_{\mathrm{eff}}^{s}+H_{\mathrm{eff}}^{p}$, with $l_{p}^{(0,-1)}=0$ and $l_{p}^{(1)} \equiv l_{p} \neq 0$ in $H_{\mathrm{eff}}^{p}$ [Eq. (10)]. Specifically, we consider a fixed small negative $a_{1 D}$ (strong $s$-wave repulsion) and a weak tunable $l_{p}$ to study how these magnetic orderings occur in a trapped system with a given spin number of fermions.

Ferromagnetic vs antiferromagnetic ordering. In the $p$ wave-dominated regime, i.e., $\left|l_{p} / a_{1 D}\right| \gg 1$, the system can exhibit two types of magnetic orders depending on the sign of $l_{p}$ :

(I) $F M$ for $l_{p}<0$ : For attractive $p$-wave interaction, spin $\uparrow$ atoms tend to line up in a region with a larger particle (charge) density due to the ferromagnetic ordering resulting from the exchange coupling $J_{l}$. Thus for a harmonic confinement, the spin $\uparrow$ atoms occupy the trap center whereas the spin $\downarrow$ atoms are repelled to the edges, as shown by Fig. 2 (a1,b1). Here the attractive $p$-wave interaction within one species of fermions facilitates the formation of ferromagnetic domains with full spin segregation, i.e., a realization of itinerant FM.

(II) Néel AFM for $l_{p}>0$ : For repulsive $p$-wave interaction, spin $\uparrow$ atoms tend to be separated alternately by spin $\downarrow$ atoms. This is a necessary condition for forming the Néel AFM. Indeed, for system with $N_{\uparrow}=N_{\downarrow}+1$, the ground state has the unique Néel AFM spin order $|\{\uparrow \downarrow \ldots \uparrow \downarrow \uparrow\}\rangle$, as shown in Fig. 2 (b3). Meanwhile, we note that for a system with other spin numbers, the ground state of $H_{\mathrm{eff}}^{p}$ could be degenerate. In particular, for equal mixture $N_{\uparrow}=N_{\downarrow}=N / 2$, there is $(N / 2+1)$-fold of degeneracy. For instance, for $N_{\uparrow}=N_{\downarrow}=2$, they are $|\{\uparrow \downarrow \uparrow \downarrow\}\rangle,|\{\uparrow \downarrow \downarrow \uparrow\}\rangle,|\{\downarrow \uparrow \downarrow \uparrow\}\rangle$. Such degeneracy can be lifted by the presence of $H_{\text {eff }}^{s}$, leading to a unique ground state with the largest weight in $|\{\uparrow \downarrow \downarrow \uparrow\}\rangle$. The Néel $\mathrm{AFM}$ is quite prominent in the resulting spin distribution, especially in the outer wings of the trap, as shown by Fig. 2 (a3) for $N_{\uparrow}=N_{\downarrow}=6$. Though we cannot enumerate for all specific combinations of $\left\{N_{\uparrow}, N_{\downarrow}\right\}$, we expect the Néel AFM is in general the most dominated magnetic order for repulsive $p$-wave interaction.

In Fig. 2, we show the spin density distribution $\rho_{\sigma}(x)=$ $\sum_{i} n_{i \sigma} \rho_{i}(x)$, with $n_{i \sigma}$ the density of spin $\sigma$ at site $i$ obtained from the effective spin-chain Hamiltonian and $\rho_{i}(x)$ the particle density at order $i$ in 1D traps [11,12,25,28]. We can see evident spin configuration of FM or Néel AFM being developed as increasing $\left|l_{p} / a_{1 D}\right|$ from the $s$-wave-dominated $\left[l_{p}=0\right.$ in Fig. $\left.2(\mathrm{a} 2, \mathrm{~b} 2)\right]$ to the $p$-wave-dominated $\left[\left|l_{p} / a_{1 D}\right|=\right.$ 3 in Fig. 2 (a1,a3;b1,b3)] regime.

We note that the emergence of the above magnetic orders is a crossover rather than a sharp phase transition. This can be seen from Fig. 3 (a1,b1), that the ground-state energy evolves continuously as varying $l_{p}$. It is useful to give an estimation to the crossover location, at which point the system is expected to enter the $p$-wave-dominated regime and develop an explicit feature of the FM or Néel AFM order in space. In order to do that, we compare two energies: $E_{s / p}=\left\langle\psi_{s / p}\left|H_{\mathrm{eff}}\right| \psi_{s / p}\right\rangle$, 

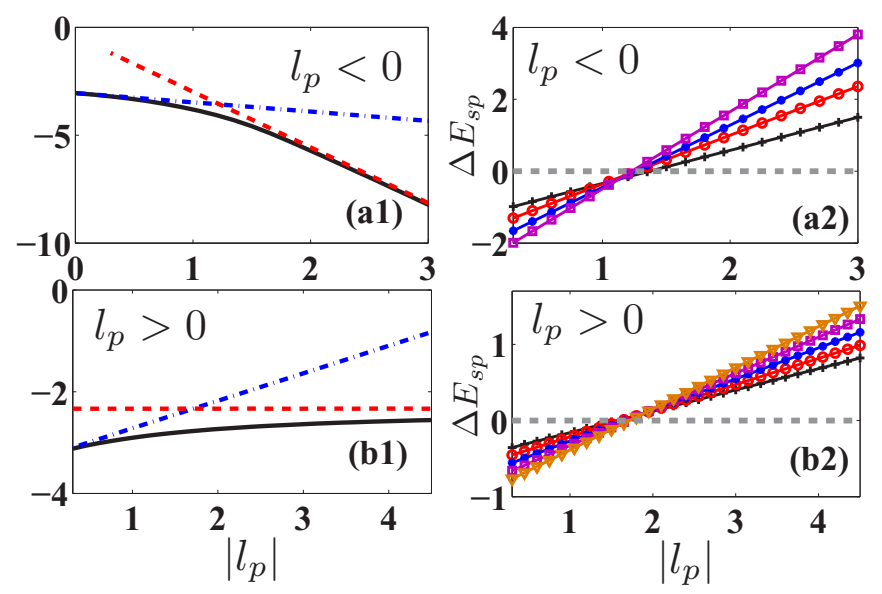

FIG. 3. Crossover from $s$-wave to $p$-wave-dominated regime. (a1,b1): The energies $E$ (solid), $E_{s}$ (dash-dot) and $E_{p}$ (dash) as a function of $\left|l_{p}\right|$. (a1) is for a $6 \uparrow+6 \downarrow$ system with $l_{p}<0$; (b1) is for a $7 \uparrow+6 \downarrow$ system with $l_{p}>0$. (a2,b2): $\Delta E_{s p}=E_{s}-E_{p}$ as a function of $\left|l_{p}\right|$. In (a2), $l_{p}<0$, and the crossing, circle, diamond, and square points, respectively, are for $N_{\uparrow}=N_{\downarrow}=3,4,5,6$; in (b2), $l_{p}>0$, and the crossing, circle, diamond, square, and triangle points, respectively, are for $N_{\uparrow}=N_{\downarrow}+1=3,4,5,6,7$. In all plots, the energy unit is $-m a_{1 D} J_{1} N / 4$, and $\left|l_{p}\right|$ is in units of $-a_{1 D}(>0)$.

where $\psi_{s}$ and $\psi_{p}$, respectively, are the ground states of $H_{\mathrm{eff}}^{s}$ and $H_{\mathrm{eff}}^{p}$ only, representing the states in the $s$-wave and $p$ wave-dominated regimes. The crossover value of $l_{p}$ is then determined by matching $E_{s}$ with $E_{p}$. In Fig. 3 (a2,b2), we plot the energy difference $\Delta E_{s p}=E_{s}-E_{p}$ as a function of $l_{p} /\left(-a_{1 D}\right)$ for different particle numbers $N$. It is found that the crossover to FM occurs at $l_{p} \approx-1.3\left|a_{1 D}\right|$ for equal mixtures up to $N=12$, and to AFM it occurs at $l_{p} \approx 2\left|a_{1 D}\right|$ for fermions with $N_{\uparrow}-N_{\downarrow}=1$ up to $N=13$. Therefore these orders can be easily achieved with weak $p$-wave interactions given the system is in the strong-coupling regime with very small $a_{1 D}$.

We remark on several unique advantages for the present scheme to engineer quantum magnetisms in ultracold atoms. First, the required conditions, controlled by a single interaction parameter $l_{p} / a_{1 D}$, are practically achievable in current experiments. Second, the resulting ground state is nondegenerate with a robust spin configuration (against external perturbations), thanks to the particular $p$-wave interaction in ${ }^{40} \mathrm{~K}$ and the presence of external trapping potential. Third, one can conveniently achieve distinct magnetic orderings, the Néel AFM and the itinerant FM, in the same system, simply by changing the sign of the $p$-wave interaction at different sides of the resonance. Finally, since the weak $p$-wave interaction will not alter the fermionalized nature of the $1 \mathrm{D}$ system, the atom loss is expected to be essentially suppressed as measured in the hard-core bosons [32], and thus the stability of the system can be guaranteed.

Experimental relevance. The system described above can be realized using the ${ }^{40} \mathrm{~K}$ atoms with two hyperfine states $\left|F=9 / 2, m_{F}=-7 / 2\right\rangle \equiv|\uparrow\rangle$ and $\left|F=9 / 2, m_{F}=-9 / 2\right\rangle \equiv$ $|\downarrow\rangle$. An $s$-wave Feshbach resonance occurs between $|\uparrow\rangle$ and $|\downarrow\rangle$ at magnetic field $202.1 \mathrm{G}$ (with width $8 \mathrm{G}$ ), very close to the splitted $p$-wave resonances at $198.8 \mathrm{G}$ and $198.3 \mathrm{G}$ between two $|\uparrow\rangle$ states [38]. Near the $p$-wave resonances, the $s$-wave scattering length $a_{s} \sim 30 \mathrm{~nm}$, which can lead to the strong interaction in quasi-1D geometry through the confinement-induced resonance with transverse confinement length of the same order of $a_{s}$ [44]. This is achievable by applying deep optical lattices with the confinement length of each harmonic well about one-tenth of the lattice spacing, $\sim 400 \mathrm{~nm}$. Alternatively, the strong interaction can also be achieved by making the system sufficiently dilute such that $\left|a_{1 D}\right|$ is much smaller than the interparticle distance. For the $p$-wave interaction, as the $p$-wave Feshbach resonances and its induced 1D resonances [45-47] are close to each other and all of them have a resonance width of about $0.5 \mathrm{G}$, a weak $p$-wave interaction can then be adjusted by fine-tuning the magnetic field around these resonances (with resolution $\lesssim 10 \mathrm{mG}$ [48]). The predicted FM and Néel AFM order can be probed by imaging the spin density for a many-body system with over hundreds of fermions, or by the tunneling and singleparticle level measurements for small cluster systems as in Ref. [37].

Final remark. Our results shows a powerful realization of intriguing magnetic orderings in a trapped geometry of ultracold atomic gases with interactions in multiple partial-wave scattering channels. In particular, the inclusion of anisotropic $p$-wave interactions leads to an effective Heisenberg $X X Z$ spin chain with much richer magnetisms as compared to the pure $s$-wave interacting case. These results also shed light on the quantum magnetism of fermion systems in higher dimensions. For instance, tuning on a $p$-wave attraction between one species of fermions will hopefully enhance the ferromagnetic correlation in the repulsive upper branch of a 2D or 3D spin-1/2 Fermi gas, which could be detectable via a pronounced signal of spin fluctuation (as measured in Ref. [5]) before the atom losses dominate.

Acknowledgments. This work is supported by the National Natural Science Foundation of China (NNSFC) under Grants No. 11374177, No. 11421092, and No. 11374331, and by key NNSFC Grant No. 11534014, by the National Basic Research Program of China under Grant No. 2012CB922101, and the programs of the Chinese Academy of Sciences. X.W.G. and X.C. thank Y-Z. Jiang, D. Kurlov, G. Shlyapnikov, and Y.-P. Wang for helpful discussions.
[1] A. Auerbach, Interacting Electrons and Quantum Magnetism (Springer, New York, 1994).

[2] D. Greif, T. Uehlinger, G. Jotzu, L. Tarruell, and T. Esslinger, Science 340, 1307 (2013).
[3] R. A. Hart, P. M. Duarte, T.-L. Yang, X. Liu, T. Paiva, E. Khatami, R. T. Scalettar, N. Trivedi, D. A. Huse, and R. G. Hulet, Nature (London) 519, 211 (2015). 
[4] G.-B. Jo, Y.-R. Lee, J.-H. Choi, C. A. Christensen, T. H. Kim, J. H. Thywissen, D. E. Pritchard, and W. Ketterle, Science 325, 1521 (2009).

[5] C. Sanner, E. J. Su, W. Huang, A. Keshet, J. Gillen, and W. Ketterle, Phys. Rev. Lett. 108, 240404 (2012).

[6] C. W. von Keyserlingk and G. J. Conduit, Phys. Rev. A 83, 053625 (2011).

[7] X. Cui and T.-L. Ho, Phys. Rev. Lett. 110, 165302 (2013).

[8] E. Fratini and S. Pilati, Phys. Rev. A 90, 023605 (2014).

[9] D. Pecak, M. Gajda, and T. Sowinski, New J. Phys. 18, 013030 (2016).

[10] A. S. Dehkharghani, A. G. Volosniev, and N. T. Zinner, J. Phys. B: At. Mol. Opt. Phys. 49085301 (2016).

[11] X. Cui and T.-L. Ho, Phys. Rev. A 89, 023611 (2014).

[12] F. Deuretzbacher, D. Becker, J. Bjerlin, S. M. Reimann, and L. Santos, Phys. Rev. A 90, 013611 (2014).

[13] L. Yang, L. Guan, and H. Pu, Phys. Rev. A 91, 043634 (2015).

[14] D. Zhang, W. Chen, and H. Zhai, arXiv:1510.08303.

[15] M. A. Cazalilla, A. F. Ho, and M. Ueda, New J. Phys. 11, 103033 (2009).

[16] P. Massignan, J. Levinsen, and M. M. Parish, Phys. Rev. Lett. 115, 247202 (2015).

[17] Y. Li, E. H. Lieb, and C. Wu, Phys. Rev. Lett. 112, 217201 (2014); S. Xu, Y. Li, and C. Wu, Phys. Rev. X 5, 021032 (2015).

[18] X.-W. Guan, M. T. Batchelor, and C. Lee, Rev. Mod. Phys. 85, 1633 (2013).

[19] X.-W. Guan, M. T. Batchelor, and M. Takahashi, Phys. Rev. A 76, 043617 (2007).

[20] K. A. Matveev and A. Furusaki, Phys. Rev. Lett. 101, 170403 (2008).

[21] X.-W. Guan, M. T. Batchelor, and J.-Y. Lee, Phys. Rev. A 78, 023621 (2008).

[22] A. G. Volosniev, D. V. Fedorov, A. S. Jensen, M. Valiente, and N. T. Zinner, Nat. Commun. 5, 5300 (2014).

[23] J. Levinsen, P. Massignan, G. M. Bruun, and M. M. Parish, Sci. Adv. 1, e1500197 (2015).

[24] A. G. Volosniev, D. Petrosyan, M. Valiente, D. V. Fedorov, A. S. Jensen, and N. T. Zinner, Phys. Rev. A 91, 023620 (2015).

[25] L. Yang and X. Cui, Phys. Rev. A 93, 013617 (2016).

[26] M. D. Girardeau, J. Math. Phys. 1, 516 (1960).

[27] M. D. Girardeau, and A. Minguzzi, Phys. Rev. Lett. 99, 230402 (2007).

[28] F. Deuretzbacher, K. Fredenhagen, D. Becker, K. Bongs, K. Sengstock, and D. Pfannkuche, Phys. Rev. Lett. 100, 160405 (2008).
[29] L. Guan, S. Chen, Y. Wang, and Z.-Q. Ma, Phys. Rev. Lett. 102, 160402 (2009).

[30] The quantum magnetisms in this system have a different mechanism from those in real 1D lattices, where the nextnearest-neighbor hopping or interaction plays a crucial role, see S. Daul and R. M. Noack, Phys. Rev. B 58, 2635 (1998); K. Yang, Phys. Rev. Lett. 93, 066401 (2004).

[31] D. M. Gangardt and G. V. Shlyapnikov, Phys. Rev. Lett. 90, 010401 (2003).

[32] E. Haller, M. Rabie, M. J. Mark, J. G. Danzl, R. Hart, K. Lauber, G. Pupillo, and H.-C. Nagerl, Phys. Rev. Lett. 107, 230404 (2011).

[33] S. E. Gharashi and D. Blume, Phys. Rev. Lett. 111, 045302 (2013).

[34] P. O. Bugnion and G. J. Conduit, Phys. Rev. A 87, 060502(R) (2013).

[35] T. Sowinski, T. Grass, O. Dutta, and M. Lewenstein, Phys. Rev. A 88, 033607 (2013).

[36] E. J. Lindgren, J. Rotureau, C. Forssen, A. G. Volosniev and N. T. Zinner, New J. Phys. 16, 063003 (2014).

[37] S. Murmann, F. Deuretzbacher, G. Zurn, J. Bjerlin, S. M. Reimann, L. Santos, T. Lompe, and S. Jochim, Phys. Rev. Lett. 115, 215301 (2015).

[38] C. A. Regal, C. Ticknor, J. L. Bohn, and D. S. Jin, Phys. Rev. Lett. 90, 053201 (2003); C. Ticknor, C. A. Regal, D. S. Jin, and J. L. Bohn, Phys. Rev. A 69, 042712 (2004).

[39] Such $p$-wave potential works only for weak interaction, giving the Hartree-Fork interaction energy. In the strong-coupling regime, the interaction $U_{p}$ needs to be renormalized, see $\mathrm{X}$. Cui, arXiv:1605.04363.

[40] C. N. Yang, Phys. Rev. Lett. 19, 1312 (1967).

[41] Y.-Z. Jiang, D. V. Kurlov, X.-W. Guan, and G. V. Shlyapnikov, arXiv:1512.01392.

[42] T. Cheon and T. Shigehara, Phys. Rev. Lett. 82, 2536 (1999).

[43] M. Takahashi, Thermodynamics of One-Dimensional Solvable Models (Cambridge University Press, Cambridge, UK, 1999).

[44] M. Olshanii, Phys. Rev. Lett. 81, 938 (1998).

[45] B. E. Granger and D. Blume, Phys. Rev. Lett. 92, 133202 (2004).

[46] L. Pricoupenko, Phys. Rev. Lett. 100, 170404 (2008).

[47] S.-G. Peng, S. Tan, and K. Jiang, Phys. Rev. Lett. 112, 250401 (2014).

[48] C. Luciuk, S. Trotzky, S. Smale, Z. Yu, S. Zhang, J. H. Thywissen, Nature Phys. (2016), doi:10.1038/nphys3670. 